\title{
Parámetros de la fuente y del movimiento del suelo del terremoto de Lorca de 2011
}

\author{
GERARDo AlguACIL DE LA BLANCA ${ }^{1,2}$, FrANCisCO VidAL SÁNCHEZ ${ }^{1,2}$, \\ DANIEL STICH ${ }^{1,2}$, FLOR DE LIS MANCILLA PÉREZ ${ }^{1,2}$, JOSÉ ANGEL LÓPEZ COMINO ${ }^{1,2}$, \\ José MORALES SOTO ${ }^{1,2} \&$ MANUEL NAVARRO BERNAL ${ }^{1,3}$ \\ ${ }^{1}$ Instituto Andaluz de Geofísica, Universidad de Granada \\ ${ }^{2}$ Dpto. de Física Teórica y del Cosmos, Universidad de Granada \\ ${ }^{3}$ Dpto. de Física Aplicada, Universidad de Almería \\ alguacil@iag.ugr.es
}

Recibido: 22/06/2012

Aceptado: 09/10/2012

\section{Resumen}

Se han relocalizado 113 eventos de la serie sísmica de Lorca, con datos de redes sísmicas temporales y permanentes, mediante el algoritmo de la doble diferencia. Las réplicas de los primeros días se distribuyen espacialmente con una dimensión de unos $5 \mathrm{~km}$ de largo, con rumbo NE-SW, y situadas al suroeste del evento principal, lo que sugiere una propagación de la ruptura hacia el SW, a lo largo de la falla de Alhama de Murcia. Se ha obtenido un mecanismo similar para los tres sismos mayores, con un fallamiento oblicuo, predominantemente inverso y se han estimado los parámetros de la fuente de estos tres terremotos Se ha simulado el movimiento horizontal del suelo en 11 puntos de Lorca cuya estructura local es conocida por experimentos de SPAC. Los valores obtenidos de una serie de parámetros del movimiento del suelo ( $P G A, P G V, A I, C A V, S I, S A$ y SV) son mayores en los 11 puntos que los de la estación LOR y también mayores que los valores medios obtenidos para sismos euro-mediterráneos de intensidad local VIII (EMS). Sin embargo, los $S_{D}$ son excepcionalmente cortos y menores que los valores de referencia. Los valores más altos de los espectros de respuesta de aceleración y de velocidad se dan para periodos menores de $0.7 \mathrm{~s}$, con máximos de aceleración espectral en $0.15 \mathrm{~s}$ y de velocidad en $0.5 \mathrm{~s}$. El espectro de energía de entrada está bien relacionado con la destructividad de las sacudidas sísmicas en cada lugar. Velocidades equivalentes $>60 \mathrm{~cm} / \mathrm{s}$ se han obtenido en casi todos los sitios y $>100 \mathrm{~cm} / \mathrm{s}$ (para periodos 0.3-0.6 s) en algunos. Los factores de cercanía y las características del mecanismo focal y de la respuesta del terreno explican los valores altos obtenidos de parámetros del movimiento del suelo en Lorca y muestran la gran influencia de la fuente y del sitio en las características del movimiento fuerte en las cercanías de la ruptura.

Palabras clave: Mecanismo focal, parámetros del movimiento del terreno, intensidad instrumental, energía sísmica de entrada.

\section{Source and ground-motion parameters of the 2011 Lorca earthquake}

\begin{abstract}
113 events of the Lorca seismic series has been relocated by using Double difference algorithm and data from both temporary and permanent seismic networks. Relocations yield shallow hypocentral distribution of aftershocks with a $\sim 5 \mathrm{~km}$ long, NE-SW trending, placed SW of the mainshock, suggesting a SW propagating rupture along the Alhama de Murcia fault. Similar oblique reverse faulting mechanism has been obtained for three largest events. Source parameters of these three
\end{abstract}


earthquakes have been estimated. Horizontal ground motion was estimated at 11 city points whose local structure was known by SPAC experiments. A set of ground motion parameters (PGA, PGV, AI, CAV, SI, SA and SV) here calculated, have higher values at these points respect to the ones at LOR station. All parameter values are also above the expected values for Euro -Mediterranean earthquakes with local intensity VIII (EMS). Nevertheless, SD values are unusually short and less than the reference ones. Higher values of the response spectra of acceleration and velocity are given for periods of less than $0.7 \mathrm{~s}$, with maximum spectral acceleration at $0.15 \mathrm{~s}$ and velocity at $0.5 \mathrm{~s}$. The elastic input energy spectrum is well connected to the shake destructiveness in each place. Equivalent velocity $>60 \mathrm{~cm} / \mathrm{s}$ is found in almost all sites and $>100 \mathrm{~cm} / \mathrm{s}$ (for periods 0.3 to $0.6 \mathrm{~s}$ ) in someone. Factors such as proximity, and focal mechanism and ground response characteristics explain the high ground motion parameter values obtained in Lorca sites and show the great influence of the source and site conditions on the characteristics of strong ground motion in the vicinity of the rupture.

Keywords: Focal mechanism, ground-motion parameters, instrumental intensity, input energy spectrum.

Sumario: Introducción. 1. Estructura geológica local del área urbana de Lorca y periodos predominantes del suelo. 2. Tensor momento sísmico de los tres sismos más importantes de la serie. 3 . Relocalización con el método de doble diferencia. 4. Parámetros del movimiento fuerte del terreno. 4.1. Parámetros cinemáticos del movimiento del suelo. 4.2. Parámetros relacionados con la energía. 4.3. Parámetros espectrales. 5. Análisis y discusión de resultados. 6. Conclusiones. Agradecimientos. Referencias bibliográficas.

\section{Referencia normalizada}

Alguacil1, G., Vidal, F., Stich, D., Mancilla, F., López, J.A., Morales, J., Navarro, M. (2012). Parámetros de la fuente y del movimiento del suelo del terremoto de Lorca de 2011. Física de la Tierra, Vol.24, 41-69.

\section{Introducción}

Los recientes terremotos de Lorca de 2011 han mostrado que, en la región cercana a la fuente, sismos pequeños, como los ocurridos el 11 de Mayo, pueden provocar movimientos sísmicos severos no solo en suelos blandos sino también en roca. La estación LOR, situada en la parte norte del centro de la ciudad, registró en el sismo principal ( $\mathrm{Mw}$ 5.2) unos valores máximos de aceleración $\left(\mathrm{PGA}^{1}\right)$ y de velocidad (PGV) de $0.37 \mathrm{~g}$ y $0.35 \mathrm{~m} / \mathrm{s}$, respectivamente. Estos valores del movimiento del terreno catalogan a la sacudida como severa, según los criterios de clasificación de Shake-Map (Wald et al., 1999), y corresponden a la parte inferior de valores esperables para una intensidad de grado VIII (escala MM). El evento precursor ( $\mathrm{Mw}$ 4.6) y la réplica principal (Mw 3.9) también tuvieron valores altos de PGA y de PGV, a pesar de su magnitud pequeña, causando el primero daños apreciables en estructuras rígidas. Estos valores máximos de la sacudida sísmica pueden ser razonablemente explicados por la proximidad del epicentro $(<5 \mathrm{~km})$,

\footnotetext{
${ }^{1}$ Las siglas de los parámetros utilizadas aquí se corresponden con sus nombres en inglés por su amplio uso en la literatura internacional especializada.
} 
la poca profundidad de la ruptura $(4,6 \mathrm{~km})$ y los efectos de directividad, ya que la propagación del frente de ruptura y la dirección de deslizamiento están en dirección a Lorca (López-Comino et al., 2012).

Tanto el terremoto premonitorio (15h:05m), el terremoto principal (16h:47) como la serie de réplicas fueron ampliamente registrados durante las semanas siguientes, gracias a una extensa red de estaciones sísmicas desplegadas en la región. Estas estaciones gestionadas por diferentes organismos estaba conformada por: estaciones permanentes de banda ancha (IGN, IAG, ROA/UCM/Geofon y IGN/UA), por estaciones temporales de banda ancha desplegadas durante el proyecto INDALO y pertenecientes al IAG, estaciones de movimiento fuerte de la red de acelerógrafos del IGN y por una estación de banda ancha de despliegue rápido. Esta estación se instaló en la propia ciudad de Lorca a las pocas horas de ocurrido el terremoto principal.

Uno de los aspectos que llama la atención de la serie temporal es su corta duración y el escaso número de réplicas comparado con otras series sísmicas similares acaecidas en la región, como son la de Bullas 2002 y la de La Paca 2005. La serie de Lorca 2011 apenas duró un mes, donde se contabilizaron 143 terremotos (a 19 de Julio de 2011), de los cuales el 80\% ocurrieron durante la primera semana. Para las series anteriormente mencionadas, el número de réplicas se elevaron a 290 y 605 terremotos para Bullas y La Paca respectivamente. También es interesante la distribución de las magnitudes; analizando el parámetro b de la ley de Gutemberg -Richter se observa un valor muy bajo, 0.6 (Morales et al., 2012), aunque similar al observado por Mezcua et al (1984) para la serie de Lorca de 1977.

Las primeras localizaciones rutinarias de la serie sísmica realizadas por el IGN y el IAG, tomando datos principalmente de sus respectivas redes permanentes, mostraban que lejos de agruparse las réplicas en torno al terremoto principal, estas se distribuían en el valle del Guadalentín sin un patrón determinado, varios kilómetros al este de la zona fuente (Figura 1A). Esto evidenciaba un posible efecto de falta de datos en la cercanía de la zona fuente, que se vió corroborado cuando se incorporaron los datos provenientes de las redes temporales del proyecto INDALO (del IAG), los datos de movimiento fuerte de los acelerógrafos de la zona epicentral y la estación temporal instalada en la ciudad de Lorca por el IGN el 12 de Mayo (Figura 1B). Hemos utilizado datos de lecturas de terremotos registrados hasta el 7 de Julio de 2011, con un total de 113 eventos localizados de los cuales el $80 \%$ ocurrieron en la primera semana de la serie. Después de un picking exhaustivo, incluyendo todas las lecturas de fases $\mathrm{P}$ y $\mathrm{S}$ disponibles en todas las estaciones, tanto permanentes como temporales, se realizó una nueva localización utilizando el modelo de tierra estándar del IAG. Esta segunda localización ya mostraba como los epicentros confluían en las cercanías de la falla de Alhama.

Otra singularidad del terremoto principal en Lorca es la desigual distribución del nivel del movimiento en diferentes zonas de la ciudad, evidenciada por la distribución de los daños. Una comparación del grado de daño y porcentaje de 
estructuras dañadas, en edificios de vulnerabilidad similar, indica diferencias entre zonas de la ciudad. Las zonas de mayor extensión del daño han sido las de depósitos recientes y períodos predominantes cercanos a los períodos naturales de los edificios (Navarro et al., 2012).
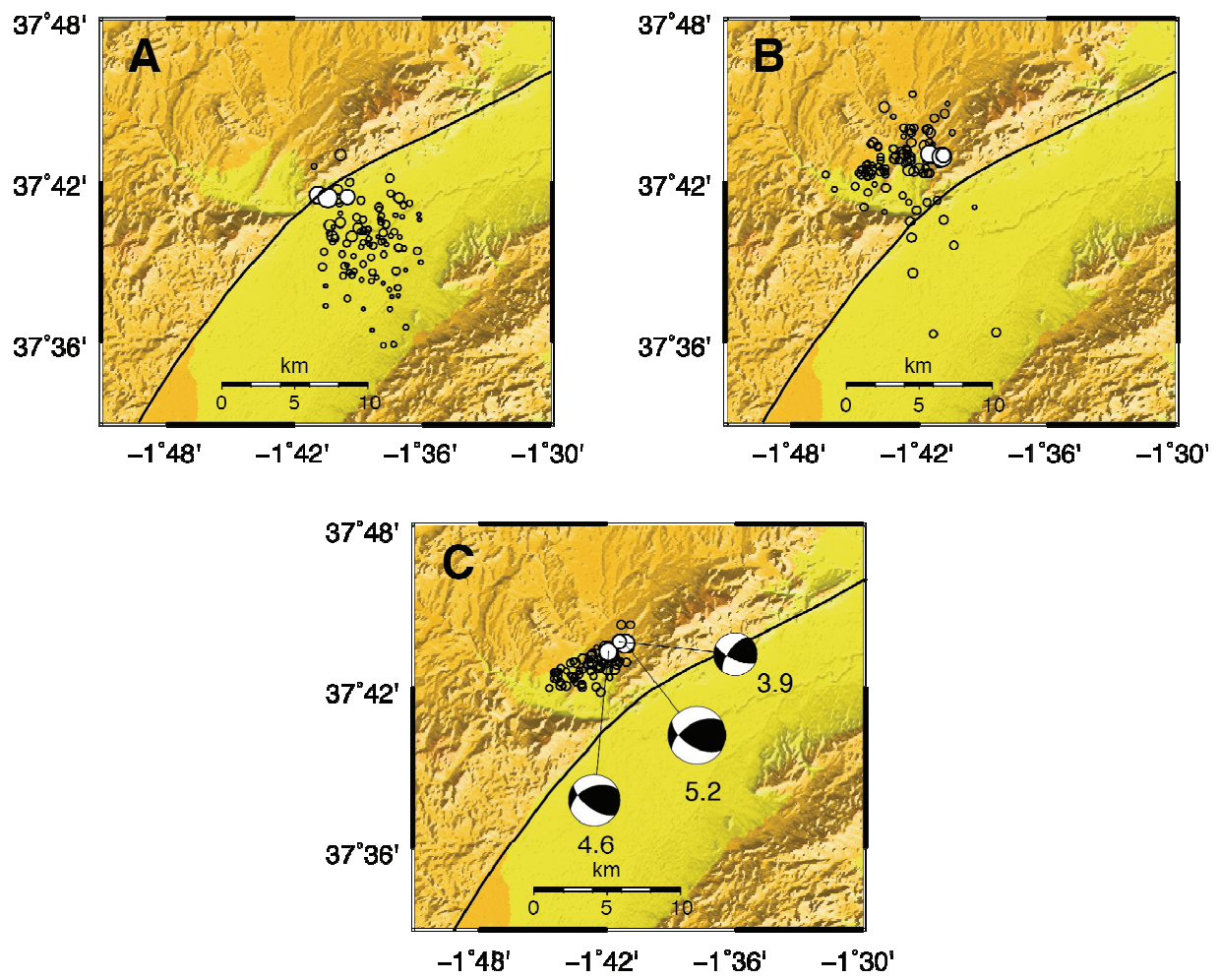

Fig. 1. Localización de la serie sísmica de Lorca 2011. Círculos blancos representan los tres eventos principales con tamaño proporcional a su magnitud. Círculos pequeños sin rellenar epicentros del resto de la serie sísmica. A) Localización epicentral inicial de rutina sin la incorporación de los datos suministrados por las redes temporales y de movimiento fuerte. B) Localización de rutina final una vez incorporados el resto de datos. C) Relocalización final de la serie sísmica completa con el método de doble diferencia y mecanismos focales de los tres eventos principales, junto a su magnitud de momento calculada mediante la inversión del tensor momento. 
Es bien sabido que los factores que influyen en los daños a construcciones ubicadas en zonas próximas a la fuente sísmica son las características de la ruptura y las condiciones geológicas locales que modifican el movimiento fuerte del terreno $\mathrm{y}$, por tanto, el de la energía sísmica de entrada en las estructuras, y finalmente la vulnerabilidad de estas. La geología superficial tiene un efecto importante en las amplitudes del movimiento fuerte. El movimiento promedio del terreno disminuye a medida que aumenta la distancia y, además, también se espera que sea mayor en sitios con valores $\mathrm{Vs}^{30}$ menores. Basándonos en el trabajo de Navarro et al (2005), las condiciones del suelo en Lorca debieron jugar un papel importante en las características de las sacudidas sísmicas y en su distribución espacial. La mayor parte de la ciudad de Lorca está construida en suelos sedimentarios, con una estructura local muy heterogénea formada por una sucesión de terrazas aluviales y sistemas de depósitos coluviales que datan desde el Plioceno hasta el presente (Alcalá et al., 2012; Navarro et al., 2012).

Valores excepcionalmente altos de PGA y PGV, respecto a los obtenidos a partir de ecuaciones de predicción de movimiento del terreno (GMPEs), se han observado en terremotos pequeños y medianos en la región cercana a la fuente (p.e. Huerta y Reinoso, 2002; Anderson et al, 2009). Anderson (2010) mostró que valores de $\mathrm{PGA} \geq 5 \mathrm{~m} / \mathrm{s}^{2}$ se registraban en terremotos con magnitudes tan bajas como $\mathrm{Mw} 4.8$ y que $\mathrm{PGV} \geq 50 \mathrm{~cm} / \mathrm{s}$ se daban en sismos con $\mathrm{Mw} \geq 5.7$ generalmente en terremotos con mecanismos de fallamiento inverso (principalmente en el bloque cabalgante) o de desgarre, sobre todo en lugares afectados por la directividad. Huerta y Reinoso (2002) mostraron que no siempre valores mayores de PGA generan mayores daños en las áreas cercanas a la fuente. Estos autores analizaron 4 sismos de magnitudes entre 5.7 y 8.1, registrados en roca a una misma distancia de la superficie de ruptura $(19 \mathrm{~km})$, que dieron valores PGA de $0.44 \mathrm{~g}(\mathrm{Mw} 5.7)$ a $0.10 \mathrm{~g}(\mathrm{Mw}$ 8.1). El sismo de mayor magnitud pero con menor PGA si produjo daños, mientras que los otros, con mayor PGA pero duraciones mucho más cortas, no produjeron daños. Así, la PGA registrada a cortas distancias del foco se correlacionaba mal con la magnitud y con el daño, destacándose la importancia de la duración de la sacudida.

Esto nos plantea el reto de ver qué características del movimiento del terreno pueden correlacionarse mejor con los daños y qué parámetros utilizables en ingeniería pueden cuantificar mejor la gravedad y el potencial de daño de las sacudidas sísmicas.

Las escalas empíricas (MM, EMS, etc.) son cualitativas y, por tanto, no establecen una medida de la intensidad sísmica (Arias, 1970) utilizable en ingeniería sísmica. Omori (1900) y Cancani (1904) fueron los primeros en intentar relacionar la intensidad con la PGA, asignando un rango de valores PGA a cada grado de sus escalas. Esta tendencia ha continuado largo tiempo, pero trabajos posteriores y recientes indican que los daños en construcciones no pueden ser bien estimados solo con la PGA, ya que otros factores como duración, amplitud, contenido en frecuencia, etc. son muy importantes en el desarrollo de los daños. 
En ingeniería sísmica generalmente se han venido utilizando parámetros del movimiento del suelo fácilmente correlacionables con las fuerzas y los desplazamientos que inducen en las estructuras, ya que estos últimos son parámetros empleados en el diseño sismorresistente. Otros métodos de diseño basados en el desempeño, que consideran el control del daño, requieren la identificación de índices de daño críticos. Estos índices de daño (parámetros ingenieriles de la demanda) se correlacionan con parámetros del movimiento del terreno. En todo caso la regla a cumplir es la dada por Uang y Bertero (1988): que la capacidad de absorber y disipar energía de la estructura sea mayor que la demanda que puede generar el sismo de diseño.

Una serie de parámetros obtenidos de los acelerogramas han querido expresar el potencial destructor del movimiento sísmico en un lugar p.e., Benioff, (1934), Gutenberg \& Richter (1942), Housner (1952), Hershberger (1956), Ambraseys $(1975,1995)$, Wald et al., (1999), pero no se ha alcanzado una formulación física general satisfactoria de esta relación. Por ello se han se han propuesto correlaciones empíricas entre uno o varios parámetros y la intensidad (p.e. Wald et al., 1999; Faenza y Michelini, 2010, Olea et al, 2011), o con la capacidad de daño (p.e. Cabañas et al.,1997, Iervolino et al., 2008).

Una característica relevante del evento principal de la serie sísmica de Lorca es la distribución desigual de los daños en Lorca como consecuencia de la distribución heterogénea de la vulnerabilidad sísmica de la construcción y de las diferencias espaciales en la severidad de sacudida sísmica dentro del área urbana. Para determinar estas diferencias de intensidad en diferentes lugares, en primer lugar se han revisado la localización y el mecanismo focal de los sismos relevantes y las características de la directividad en relación a la ciudad. En segundo lugar, tras comprobar que el registro de LOR no estuvo afectado significativamente por los efectos de sitio y corregir la orientación del acelerógrafo, se ha realizado una simulación del movimiento del suelo en 11 sitios mediante la convolución del acelerograma de LOR (registrado en terreno duro en un afloramiento del sustrato) con las funciones de transferencia 1-D en esos sitios. Estas funciones se han obtenido a partir de la estructura de velocidad de ondas $\mathrm{S}$ de las primeras capas superficiales, calculadas con la técnica de SPAC por Navarro et al. (2005, 2012). La simulación se ha hecho mediante el programa de DEGTRA (Ordaz y Montoya, 2002).

Para caracterizar instrumentalmente la gravedad del movimiento y el potencial de daño del terremoto en cada uno de los 12 sitios estudiados, y poder analizar los resultados en relación al comportamiento de los edificios afectados, se han evaluado un conjunto de parámetros clave del movimiento del suelo: los valores máximos (o pico) de movimiento fuerte ( $P G A$ y $P G V$ ), el espectro de respuesta de la aceleración $(S A)$ y de velocidad $(S V)$, la intensidad de Arias $(A I)$, la velocidad absoluta acumulada $(C A V)$, la duración significativa relativa $\left(S_{D}\right)$, la intensidad espectral de Housner $(S I)$, y el espectro de energía de entrada (IES). 


\section{Estructura geológica local del área urbana de Lorca y períodos predomi- nantes del suelo}

Dada la importancia de la posible modificación de las características de las sacudidas en diferentes lugares de la ciudad por cambios en la estructura geológica superficial, se ha tomado de Alcalá et al. (2012) un resumen las características estructurales obtenidas a partir de cartografía geológica de detalle (escala 1:10.000), datos de campo, datos geotécnicos a partir de 40 sondeos mecánicos y datos geofísicos de 27 sondeos eléctricas y de 10 perfiles de refracción sísmica superficial (con una penetración de 10 a $50 \mathrm{~m}$ ).

Esta información geológica, geotécnica y geofísica ha permitido caracterizar las propiedades mecánicas de los materiales geológicos de la ciudad y agrupar las 17 formaciones geológicas analizadas (Fig.2) en 5 formaciones geológicas, cada una con materiales con características de respuesta sísmica próximas condicionadas por los espesores. Estas formaciones son: 1) esquistos, filitas, cuarcitas y calizas dolomíticas de edad Paleozoico-Triásico de los Complejos Alpujárride y Maláguide; 2) margas, yesos y areniscas de edad Tortoniense inferior-medio y glacis consolidados del Plioceno; 3) glacis no consolidados y coluviales del Pleistoceno; 4) coluviales no consolidados y terrazas aluviales del Holoceno; 5) tierras de cultivo y rellenos antrópicos.

Los valores de $\mathrm{V}_{\mathrm{S}}$ para los diferentes materiales, obtenidos a partir de datos geotécnicos (Alcalá et al., 2012), son: materiales no consolidados del Holoceno (150-350 $\left.\mathrm{m} \mathrm{s}^{-1}\right)$, glacis del Plioceno y Pleistoceno $\left(300-500 \mathrm{~m} \mathrm{~s}^{-1}\right)$, sustrato Neógeno $\left(500-800 \mathrm{~m} \mathrm{~s}^{-1}\right)$ y sustrato pre-Triásico $\left(800 \mathrm{a}>1000 \mathrm{~m} \mathrm{~s}^{-1}\right)$. Estos valores son coherentes con los rangos de $\mathrm{V}_{\mathrm{S}}$ deducidos a partir del método SPAC. Los basamentos del Mioceno y del pre-Triásico se clasifican como terrenos medioduros y duros, respectivamente, según la NCSE-02 y el Eurocódigo EC8. Estos basamentos afloran en las zonas $\mathrm{N}$ y W de la ciudad y se han detectado entre $10 \mathrm{y}$ $50 \mathrm{~m}$ en el centro de Lorca y a más de $100 \mathrm{~m}$ hacia el sureste. El espesor de los materiales plio-cuaternarios disminuye hasta desaparecer hacia el norte y noroeste de Lorca. La estructura sedimentaria local está controlada por un conjunto de fallas de dirección ENE-WSW relacionadas con el sistema de fallas de Alhama de Murcia (FAM) y por un conjunto secundario de fallas conjugadas normales de dirección NW-SE y SW-NE que elevan o profundizan el sustrato, compartimentan la cuenca y crean así áreas con diferente espesor de materiales no consolidados.

Las campañas de medidas de ruido ambiental realizadas en la ciudad han servido para determinar, mediante el método de la razón espectral HVSR de Nakamura (1989), el período predominante del suelo en toda la ciudad y para calcular la estructura de Vs mediante el método de SPAC, utilizando arrays circulares. La distribución espacial de los períodos predominantes (Fig. 3 derecha) revela una tendencia general a aumentar de $0,1-0,3 \mathrm{~s}$, en los afloramientos del sustrato en las zonas $\mathrm{N}$ y W de la ciudad, cambiando a 0.3-1.0 s hacia las zonas central y oriental, donde afloran las formaciones plio-cuaternarias con espesor variable, siendo por lo general cada vez mayores hacia el SE (Navarro et al., 2012). Estas zonas urba- 
nas con aluviales presentan una considerable variabilidad lateral en términos de forma, tienen además múltiples picos en la razón espectral HVSR, estando el pico predominante en función de los contrastes de impedancia locales. Estas características reflejan la complejidad de la estructura superficial local.

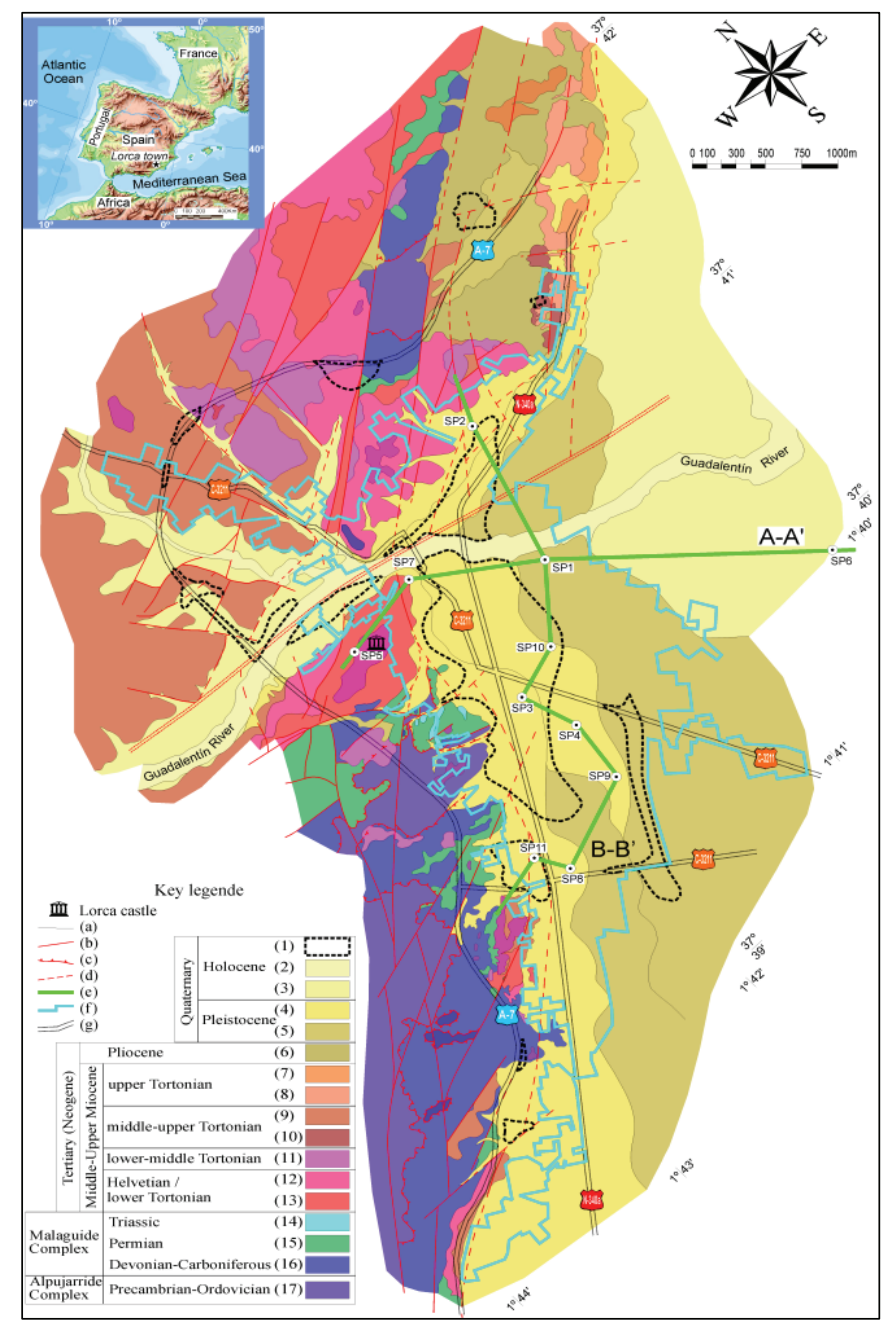

Fig. 2. Cartografía geológica de ciudad de Lorca (según Alcalá et al., 2012). Escala original 1:10.000. El cuadro adjunto describe cada material geológico. (a) contacto geológico indiferenciado; (b) falla normal; (c) falla inversa; (d) falla supuesta; (e) perfiles geológicos A-A', B-B'; (f) contorno urbano (línea azul gruesa); (g) carreteras principales. SP1 a SP11: Localización de los arrays para SPAC. 

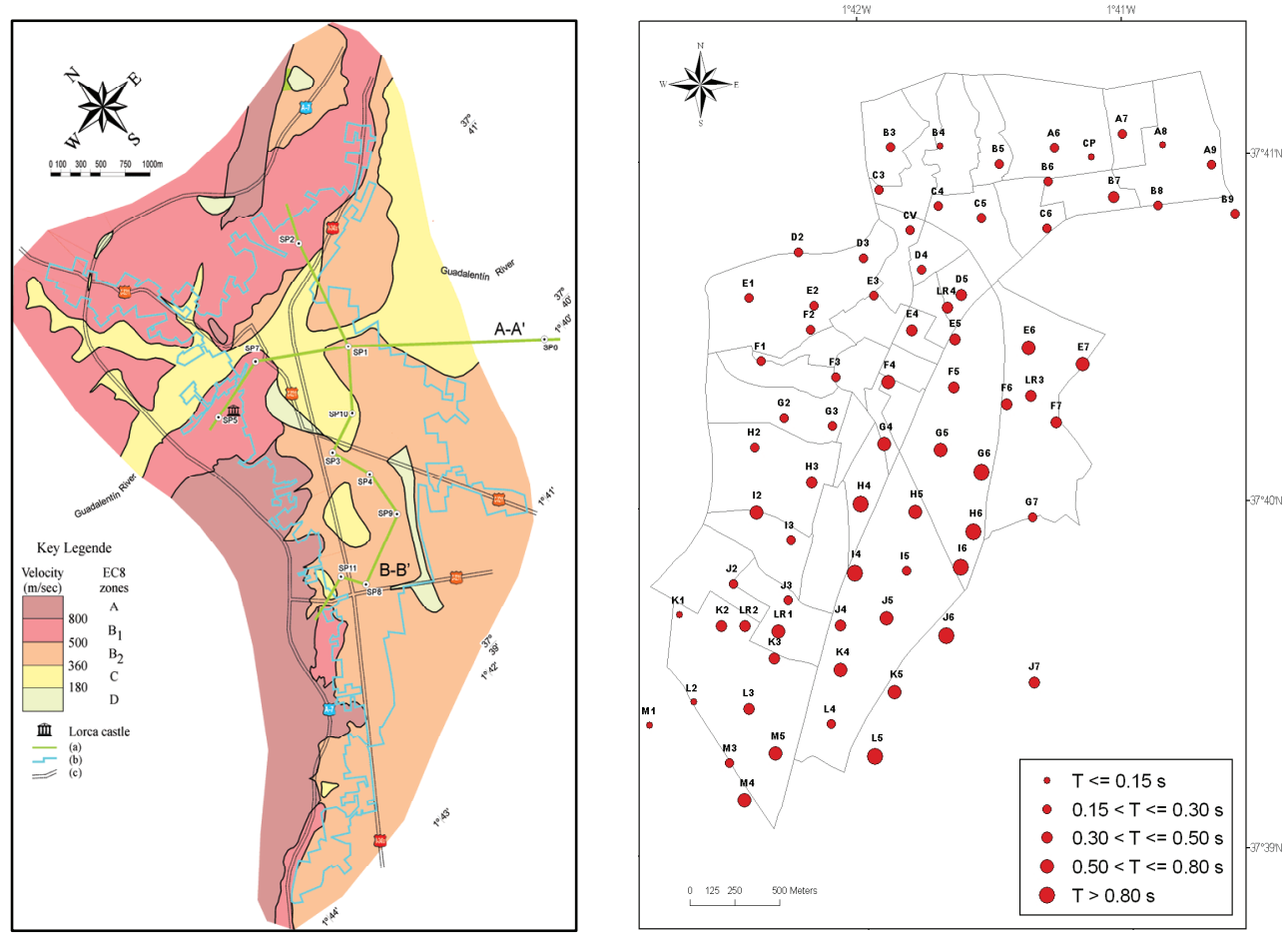

Fig. 3. Izquierda.- Clasificación del suelo de la ciudad de Lorca (Navarro et al., 2012) según el Eurocódigo EC8 (2008) y basada en la velocidad media de ondas S en los primeros $30 \mathrm{~m}\left(\mathrm{~V}_{\mathrm{S}}{ }^{30}\right.$ en $\left.\mathrm{m} / \mathrm{s}\right)$. Derecha.- Periodos predominantes del suelo obtenidos con la técnica HVSR.

Las estructuras geológicas existentes en la ciudad de Lorca se han clasificado según el Eurocódigo 8 (EC8, 2008) según los valores $\mathrm{V}_{\mathrm{S}}{ }^{30}$ obtenidos de las campañas de SPAC, que han considerado los valores $\mathrm{V}_{\mathrm{S}}$ de los perfiles de refracción de poca profundidad (IGME, 1992), los $\mathrm{V}_{\mathrm{S}}$ deducidos de valores de $\mathrm{N}$ de SPT y el espesor de las formaciones geológicas (Fig.3 izquierda).

\section{Tensor momento de los tres sismos más importantes de la serie}

Poco después de ocurrido el sismo principal se obtuvieron soluciones rápidas del tensor momento, las cuales se mostraron en las direcciones web de diferentes organismos (IGN, www.ign.es; Proyecto Global CMT, www.globalcmt.org; USGS / SLU, www.usgs.gov; GeoAzur, www.geoazur.oca.eu; GFZ, www.geofon.gfz-potsdam.de; INGV, www.ingv.it). En todos los casos, los resultados obtenidos compartían una solución de falla oblicua de régimen inverso, con uno de los planos nodales en la dirección SW-NE. La localización preliminar 
junto a la ciudad de Lorca y una solución del mecanismo focal coincidente con la dirección de la falla de Alhama de Murcia fue un punto clave para relacionar rápidamente este terremoto con la FAM (Vissers y Meijninger, 2011).

De la serie sísmica completa, sólo los sismogramas regionales de banda ancha de los tres eventos principales eran susceptibles para llevar a cabo un esquema de inversión, en el dominio del tiempo, que permitiera recuperar la información de los parámetros de la fuente sísmica mediante la inversión del tensor momento sísmico regional. El aumento y la mejora de la instrumentación así como la cobertura de las redes sísmicas permanentes y temporales gestionadas por los diferentes organismos mencionados, ha sido clave para el registro de los tres eventos mencionados con un número amplio de sismómetros de banda ancha. De acuerdo con el procedimiento descrito en Stich et al., (2003), se realizó una inversión en el dominio del tiempo para recuperar la información sobre el momento sísmico escalar, los parámetros del fallamiento (acimut, buzamiento y deslizamiento) y la magnitud de momento Mw (Hanks y Kanamori, 1979). La solución se ha obtenido minimizando el ajuste cuando se comparan las formas de onda observada y calculada para una combinación de geometrías de fallas fundamentales (Langston et al, 1982). Las funciones de Green sintéticas se calcularon asumiendo un modelo de tierra en capas, con una corteza de naturaleza continental de $32 \mathrm{~km}$ de espesor (Stich et al., 2003). En la inversión se utilizó la forma de onda completa en el campo del desplazamiento para incluir la polaridad y la amplitud tanto de las ondas internas como de las ondas superficiales. Ambas formas de onda, observada y sintéticas, se filtraron entre $20-50 \mathrm{~s}$ para el premonitorio y el sismo principal (ambos con $\mathrm{M}>4,0$ ) y entre $15-35$ s para la réplica mayor (con $\mathrm{M}<4,0$ ).

Hemos utilizado en el procedimiento de inversión mencionado anteriormente una red de búsqueda en el espacio de la profundidad focal (con incrementos de 2 $\mathrm{km}$ en la corteza y $10 \mathrm{~km}$ en la parte superior del manto) para buscar la mejor profundidad del centroide y la solución del tensor de momento. Este procedimiento permite obtener una estimación independiente de la profundidad y proporciona estabilidad a la solución, reduciendo así el impacto de los errores de localización rutinaria sobre las profundidades focales estimadas del tensor momento.

En la figura $1 \mathrm{C}$ se presentan también las soluciones invertidas para los tres terremotos de mayor magnitud. En los tres casos, las soluciones muestran soluciones de fallamiento predominantemente inverso con fuerte componente de desgarre, y una orientación media NNW-SSE para los ejes de máxima presión P. La orientación del eje $\mathrm{P}$ muestra un giro contrario a las agujas del reloj respecto de la tendencia general de convergencia NO-SE entre Iberia y Nubia, pero en perfecta combinación con los resultados obtenidos por Stich et al. $(2003,2006,2010)$ para sismos localizados en la zona de cizalla de Trans-Alborán. Las soluciones para el evento principal y el premonitorio presentan prácticamente los mismos parámetros, un acimut con $\approx \mathrm{N} 240^{\circ}$ y buzamiento $\approx 55^{\circ}$ hacia el NW. La réplica es un poco diferente con un plano preferente $\mathrm{N} 216^{\circ}$ y $63^{\circ}$ de buzamiento. En todos los casos, las soluciones con plano de dirección NE-SW están claramente relacionadas con la principal falla activa en la zona: la falla de Alhama de Murcia (Martí- 
nez-Díaz, 2002; Masana et al, 2004). Para los tres casos la profundidad del centroide se fijó entre los 4 y $5 \mathrm{~km}$.

El momento sísmico escalar (Mo) y la magnitud momento (Mw) correspondientes a los tres terremotos a los que se ha podido realizar la inversión son: $\mathrm{Mo}=$ $6.5 \times 10^{16} \mathrm{Nm}(\mathrm{Mw}=5.2)$ para el sismo principal, $\mathrm{Mo}=9.6 \times 10^{15} \mathrm{Nm}(\mathrm{Mw}=4.6)$ para el precursor y $\mathrm{Mo}=7.3 \times 10^{14} \mathrm{Nm}(\mathrm{Mw}=3,9)$ para la réplica de mayor tamaño. En general, las mejores soluciones de doble-par están en concordancia con las soluciones obtenidas por Rueda et al. (2011), con pequeñas variaciones en los direcciones y buzamientos preferidos. En nuestro caso, el momento sísmico escalar es ligeramente más alto respeto al publicado por diferentes agencias en tiempo próximo (IGN, USGS, GCMT proyecto, INGV, GFZ) y por Rueda et al (2011).

\section{Relocalización con el método de doble-diferencia}

Los hipocentros de rutina obtenidos, referidos anteriormente, se han utilizado como localizaciones iniciales para obtener resultados de localización más precisos con el algoritmo estándar de doble-diferencia (Waldhauser y Ellsworth, 2000) aplicado por el programa HypoDD (Waldhauser, 2001).

En este algoritmo las diferencias entre los tiempos de viaje observado y calculado entre dos eventos, a una estación común, están relacionados con los ajustes en la localización de los hipocentros y en el tiempo origen por medio de las derivadas parciales de los tiempos de viaje de cada evento con respecto a la variable desconocida. Los residuos Doble Diferencia (DD) para pares de sismos a cada estación se minimizan utilizando el método SVD (Singular Value Descomposition), o LSQR (Conjugate Gradients Method).

La Figura 1 muestra también el resultado de la relocalización precisa con doble-diferencia. En conjunto, la distribución epicentral de los eventos de la serie se concentra en un único cluster, siguiendo una tendencia clara NE-SW que se alinea prácticamente subparalela a la dirección de la falla de Alhama de Murcia (FAM). Asociando las réplicas ocurridas en los primeros días a la ruptura del evento principal, la longitud en superficie de estas réplicas marca un segmento con unas dimensiones de aproximadamente $3.5-4 \mathrm{~km}$.

La localización del evento principal se encuentra en el borde NE del citado segmento, y separado del premonitorio aproximadamente $1 \mathrm{~km}$. Esta situación concuerda con un escenario de ruptura del evento principal hacia el SW (LópezComino et al., 2012). La réplica principal se localiza junto al evento de mayor magnitud. La distribución en profundidad del cluster evidencia una tendencia más dispersa, donde se destacan dos núcleos: el más somero se localiza entre los $0.5 \mathrm{y}$ los $2 \mathrm{~km}$ de profundidad y el principal entre los 3.5-7.5 km. Las coordenadas hipocentrales finales obtenidas mediante el algoritmo de doble diferencia para los tres eventos principales son: $37.72^{\circ} \mathrm{N},-1.70^{\circ} \mathrm{E} \mathrm{km}, 5.1 \mathrm{~km}$ para el premonitorio; $37.73^{\circ} \mathrm{N},-1.69^{\circ} \mathrm{E}, 4.6 \mathrm{~km}$ para el evento principal y $37.73^{\circ} \mathrm{N},-1.69^{\circ} \mathrm{E}, 5.5 \mathrm{~km}$ para la réplica de mayor magnitud, ocurrida también en el mismo día. 
Considerando que la distribución de las réplicas, obtenida mediante el método de doble diferencia, puede considerase directamente relacionada con la longitud de la ruptura del evento principal, se obtiene aproximadamente una longitud $\mathrm{L} \approx$ $3.5 \mathrm{~km}$. Con esta dimensión y el momento sísmico total liberado por la fuente $\left(\mathrm{Mo}=6.5 \times 10^{23}\right.$ dyn.cm), se deduce una caída de esfuerzos (Brune, 1970) entre 5885 bares, asumiendo una propagación unilateral de la fuente con un radio de la fuente entre 1.5-1.7 km. Con estos resultados no se detecta una caída de esfuerzos extremadamente elevada como principal contribución a los valores tan altos de PGA registrados en Lorca. Sin embargo un efecto de directividad, observado por Rueda et al. (2011) y López-Comino et al. (2012), a partir del análisis de la dependencia acimutal de la función fuente aparente del terremoto principal podría considerarse como un efecto adicional que explica la aceleración y los daños inusualmente elevados observados en la ciudad de Lorca.

\section{Parámetros del movimiento fuerte del terreno}

Una serie de parámetros derivados de la historia de aceleración del suelo se han propuesto en la literatura como mediciones cuantitativas de la severidad del movimiento y como indicadores del potencial de daño. Algunos de ellos, tales como la aceleración pico $(P G A)$ o los espectros de respuesta $(S A)$ son de uso estándar en los códigos sísmicos y en la práctica de ingeniería sísmica. Para caracterizar instrumentalmente la gravedad de la sacudida en 12 sitios de la ciudad, se ha calculado una serie de parámetros representativos de movimiento fuerte a partir del registro de la estación de LOR y 11 acelerogramas simulados en estos puntos. Para ello, se han utilizado las estructuras superficiales 1D, obtenidas con el método SPAC, en 11 sitios de la ciudad (Navarro et al., 2012a) y se ha calculado la respuesta de cada modelo para las ondas SH. Para obtener el acelerograma horizontal en superficie en cada punto, se ha tomado el acelerograma de la estación LOR como input en la base de cada estructura y se le ha aplicado la función de transferencia del sitio.

Los parámetros calculados son los siguientes: aceleración y velocidad pico ( $P G A, P G V$ ), intensidad Arias $(A I)$, velocidad absoluta acumulada $(C A V)$, intensidad espectral $(S I)$, duración significativa, $S_{D}$, los espectros de respuesta de aceleración, $S A$, y de velocidad, $S V$, y los espectros de energía de entrada, IES. Los valores de estos parámetros se han comparado con la media geométrica estimada (Alguacil y Vidal, 2012) para un conjunto de terremotos euro-mediterráneos de intensidades VII y VIII (escala EMS).

\subsection{Parámetros cinemáticos del movimiento del suelo}

Aceleración pico del suelo (peak ground acceleration, $\boldsymbol{P G A}$ ). El valor pico de la aceleración del suelo $(P G A)$ es un parámetro fundamental en muchos estudios de peligrosidad (p.e., Campbell, 1981; Boore \& Joyner, 1982; Margottini et al., 1992; Karim, \& Yamazaki, 2002; Ambraseys et al., 2005), y en los códigos sísmicos. 
Por ejemplo, el español (NCSE-02, 2002) usa la aceleración básica para escalar los espectros de respuesta de cálculo en cada emplazamiento.

El valor de PGA puede referirse al máximo valor del módulo de la aceleración, al máximo valor en un eje o al máximo valor en el plano horizontal (PGAH). En la mayoría de los casos, el valor pico se alcanza en las componentes horizontales y PGA es casi igual a PGAH. Excepcionalmente, especialmente en campo cercano, pueden darse aceleraciones más altas en la componente vertical (PGAZ), como p.e. en el terremoto de Christchurch de 2011 (Bradley \& Cubrinovski, 2011), aunque su impacto en la mayoría de las estructuras es menos importante que el de las PGAH, ya que todas ellas, incluso sin diseño antisísmico, están destinadas a resistir cargas verticales.

La PGA es aún el parámetro más usado para describir la intensidad y la destructividad de un terremoto en un lugar dado por su determinación simple y porque las fuerzas de inercia dependen directamente de la aceleración. Como hemos comentado al principio, los daños observados no son consistentes con las PGA, habiéndose dado multitud de ejemplos con PGA bajas y daños altos (un caso paradigmático se dio en México DF en el sismo de 1985 con solo $0.17 \mathrm{~g}$ de PGA) mientras que otros, como p.e. el del 8 de febrero de 1988 con $0.44 \mathrm{~g}$ no tuvieron daños en la zona cercana al epicentro.

La PGA se usa aún como un indicador de la intensidad sísmica, aunque su correlación con ella es más débil para intensidades altas (p.e. Wald et al., 1999), y es uno de los dos parámetros usados en EEUU para la estima rápida de los mapas de sacudida (shake map) (Wald et al., 2005).

Los valores de $P G A$ obtenidos para los 12 sitios analizados (Tabla 1) van desde $370 \mathrm{~cm} / \mathrm{s}^{2}$ (min.) en LOR hasta unos $680 \mathrm{~cm} / \mathrm{s}^{2}$ en SP6 y SP2 (máx.). Los valores de $P G A$ de los 12 lugares se han representado en la figura 4 y se han comparado con la media geométrica de $P G A$ estimada para un conjunto de terremotos europeos de intensidades VII y VIII (escala EMS).

Velocidad pico del suelo (peak ground velocity, $\boldsymbol{P G V}$ ). El efecto de este parámetro en la dinámica estructural es menos evidente. Sin embargo su uso es amplio. En algunos códigos sísmicos se emplea PGV junto con PGA para conformar espectros elásticos de respuesta esperados (Basham et al., 1985). La razón PGA/PGV puede dar una estima de las frecuencias dominantes del movimiento del suelo (Tso et al., 1992; Bommer \& Alarcón, 2006). PGV se ha mostrado como un buen indicador del potencial de daño a líneas vitales subterráneas (FEMA, 2003) y del potencial de licuefacción en suelos (p.e. Kostadinov \& Towhata, 2002). Asimismo se correlaciona bien con valores altos de la intensidad macrosísmica (Wald et al., 1999); junto con la PGA, la PGV se usa para la estimación inmediata de los shakemaps (Wald et al., 2005). Por otra parte, particularmente a raíz del terremoto de Kobe de 1995, se ha comprobado su alta correlación con el daño en estructuras de periodo intermedio (Yamazaki \& Murao, 2000).

Los valores de $P G V$ obtenidos para los 12 sitios analizados (Tabla 1) van desde $37.5 \mathrm{~cm} / \mathrm{s}$ (min.) en LOR hasta unos $60 \mathrm{~cm} / \mathrm{s}$ en SP6 y SP4 (máx.). Los valores 
de $P G V$ de los 12 lugares se han representado en la figura 4 y se han comparado con las medias geométricas de $P G V$ estimadas para un conjunto de terremotos europeos de intensidades VII y VIII (escala EMS).

Tabla 1. Valores de los parámetros en 12 sitios de Lorca y valores medios de referencia*.

\begin{tabular}{|c|c|c|c|c|c|c|}
\hline & $\begin{array}{c}\text { PGAH } \\
\mathrm{cm} / \mathrm{s}^{2}(\mathrm{~g})\end{array}$ & $\begin{array}{c}\mathrm{PGV} \\
\mathrm{cm} / \mathrm{s}\end{array}$ & $\begin{array}{c}\mathrm{AI} \\
\mathrm{cm} / \mathrm{s}\end{array}$ & $\begin{array}{c}\mathrm{S}_{\mathrm{D}} \\
\mathrm{s}\end{array}$ & $\begin{array}{c}\mathrm{CAV} \\
\mathrm{cm} / \mathrm{s}\end{array}$ & $\begin{array}{c}\mathrm{SI}_{\max } \\
\mathrm{cm} / \mathrm{s}\end{array}$ \\
\hline $\mathrm{SP} 1$ & $585(0.59)$ & 55,1 & 147 & 1,0 & 406 & 53,3 \\
\hline $\mathrm{SP} 2$ & $\mathbf{6 8 0}(\mathbf{0 . 6 9})$ & 45,5 & 186 & $\mathbf{1 , 3}$ & $\mathbf{4 8 6}$ & 43,9 \\
\hline $\mathrm{SP3}$ & $620(0.63)$ & 55,8 & 179 & 1,1 & 456 & 53,1 \\
\hline SP4 & $643(0.66)$ & $\mathbf{6 0 , 2}$ & 187 & 1,1 & 468 & $\mathbf{5 8 , 3}$ \\
\hline SP5 & $540(0.55)$ & 44,7 & 132 & 1,1 & 391 & 42,8 \\
\hline SP6 & $\mathbf{6 7 9}(\mathbf{0 . 6 9})$ & $\mathbf{5 9 , 3}$ & $\mathbf{1 9 9}$ & 1,1 & 480 & $\mathbf{5 7 , 0}$ \\
\hline SP7 & $499(0.51)$ & 41,9 & 106 & 1,2 & 344 & 39,8 \\
\hline SP8 & $547(0.56)$ & 52,6 & 145 & 1,1 & 396 & 50,3 \\
\hline SP9 & $610(0.62)$ & 55,2 & 180 & 1,1 & 461 & 54,2 \\
\hline SP10 & $656(0.67)$ & 51,0 & 173 & 1,1 & 462 & 50,0 \\
\hline SP11 & $578(0.59)$ & 43,7 & 124 & 1,1 & 378 & 45,1 \\
\hline LOR & $370(0.38)$ & 37,5 & 68,4 & 1,2 & 290 & 35,9 \\
\hline VII * & $156(0.16)$ & 8,9 & 26,5 & 7,6 & 299 & 9,5 \\
\hline VIII * & $210(0.21)$ & 10,8 & 38,3 & 4,5 & 326 & 11,0 \\
\hline
\end{tabular}

*Valores medios de sismos euro-mediterráneos de intensidades locales VII y VIII (Alguacil y Vidal, 2012).

Valores máximos en negrita y mínimos en cursiva.

\subsection{Parámetros relacionados con la energía}

Intensidad de Arias, $\mathbf{I}_{\mathbf{A}}$. Este parámetro ha tenido una gran importancia en ingeniería sísmica. Housner (1952) propuso medir el área encerrada por la curva del espectro de pseudo-velocidad de un oscilador lineal amortiguado (SDOF), llamándole intensidad espectral. Arias (1970), al comentar el trabajo de Housner (1952), indicó que los esfuerzos máximos en los elementos elásticos de un SDOF, además de ser proporcionales a $S_{v}$, también eran proporcionales a $S_{d}$ y $S_{a}$, y por tanto, que las áreas bajo estas curvas espectrales podrían también tomarse como una medida de la intensidad sísmica.

El valor en un eje $\mathrm{I}_{\text {Aii }}$ se obtuvo (Arias, 1970) como la energía almacenada por unidad de peso por una población de osciladores armónicos de frecuencias propias uniformemente distribuidas entre 0 e $\infty$ sin amortiguar en dicho eje. La intensidad de Arias puede relacionarse bien con el potencial de licuefacción y de deslizamientos de ladera (Martínez-Rueda \& Tsantali, 2008), pero es de aplicación limitada en el diseño estructural, porque sólo parece correlacionarse bien con la demanda sísmica en estructuras de periodos cortos (Travasarau et al., 2003). 

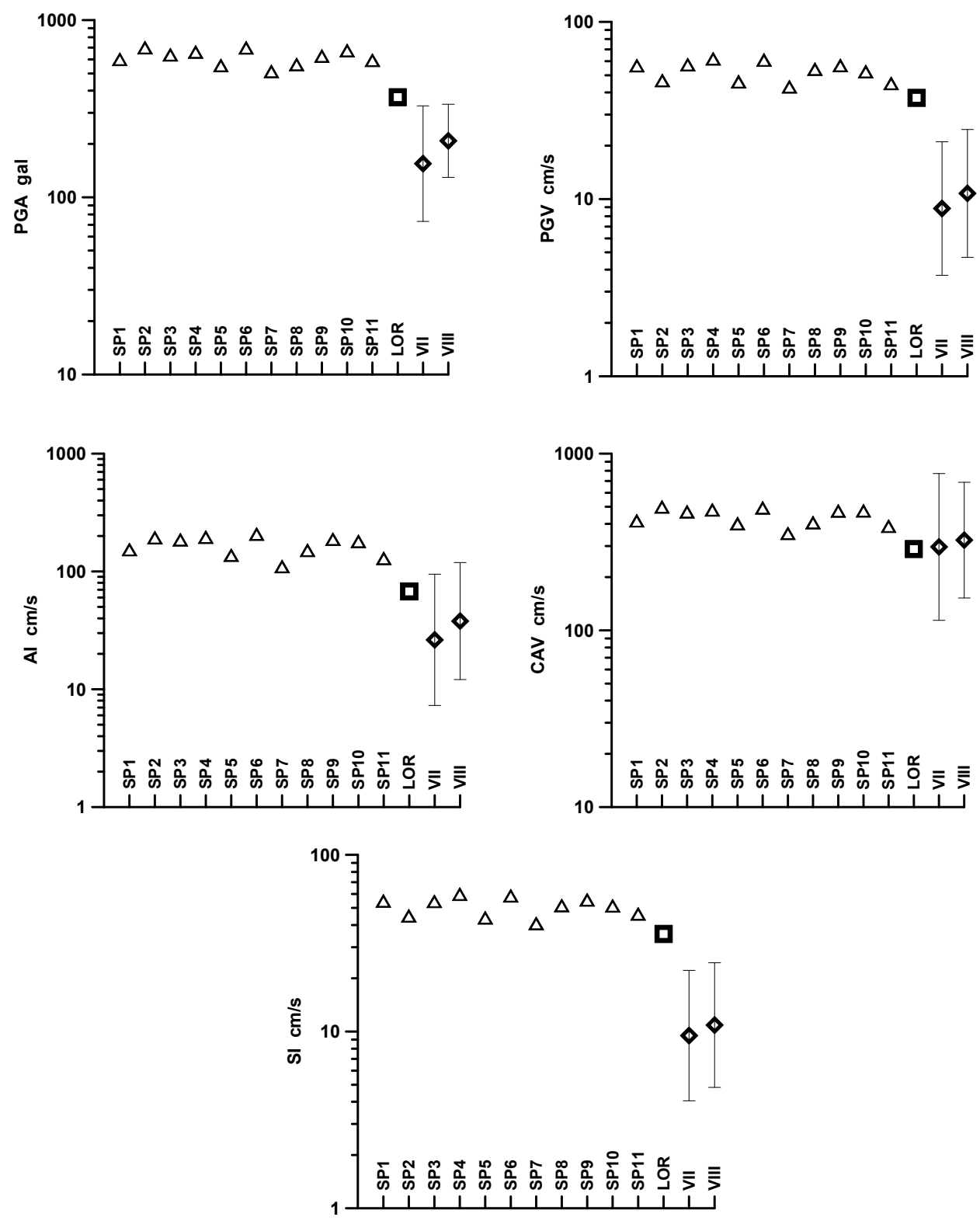

Fig. 4. Valores calculados de los parámetros instrumentales del movimiento del terreno en LOR (cuadrados) y los estimados a partir de los acelerogramas simulados en los otros 11 sitios (triángulos) de Lorca. Se han representado también la media geométrica (rombos) de cada parámetro para un conjunto de terremotos europeos de intensidades locales de VII y VIII (escala EMS). Las barras de error muestran \pm una desviación estándar. 
Arias (1970) propuso originalmente su medida de intensidad para el diseño antisísmico de centrales nucleares como un tensor simétrico.

$$
I_{A i j}=\frac{\pi}{2 g} \int_{t_{0}}^{t_{0}+D} a_{i}(t) a_{j}(t) d t
$$

donde $\mathrm{g}$ es la aceleración estándar de la gravedad $\left(\mathrm{g}=9.806 \mathrm{~m} / \mathrm{s}^{2}\right)$, a $(\mathrm{t})$ es la aceleración del suelo, y $t_{0}$ y $t_{0+D}$ son los tiempos iniciales y finales del acelerograma. En la práctica, el valor de la $\mathrm{I}_{\mathrm{A}}$ suele usarse bien en un eje $I_{A i i}$, bien el valor horizontal, $I_{A h}=I_{x x}+I_{y y}$ o el valor calculado con el módulo de $a$ al cuadrado, que corresponde a la traza del tensor,

$$
I_{A}=I_{x x}+I_{y y}+I_{z z}=\frac{\pi}{2 g} \int_{t_{0}}^{t_{0}+D}|a(t)|^{2} d t
$$

Los valores de $I_{A}$ obtenidos para los 12 sitios analizados (Tabla 1) van desde $68.4 \mathrm{~cm} / \mathrm{s}$ (min.) en LOR hasta los $199 \mathrm{~cm} / \mathrm{s}$ en SP6 (máx.). Los valores de $I_{A}$ de los 12 lugares se han representado en la figura 4 y se han comparado con las medias geométricas de $I_{A}$ estimadas para un conjunto de terremotos europeos de intensidades VII y VIII (escala EMS).

Velocidad absoluta acumulada (cumulative absolute velocity, $\boldsymbol{C A} \boldsymbol{V}$ ). Este parámetro se propuso originalmente para la definición de una acción sísmica básica operativa en centrales nucleares (EPRI, 1988) como

$$
C A V=\int_{t_{0}}^{t_{0}+D}|a(t)| d t
$$

Esto se puede interpretar como la suma de las distancias valle-pico consecutivos del registro de velocidad o como el área bajo la aceleración absoluta frente a la duración. En este sentido, tanto la amplitud de la aceleración como la duración contribuyen a este parámetro (Cabañas et al., 1997). En la definición actual (EPRI, 1991), se trata de eliminar aquellos registros de larga duración pero con aceleraciones bajas. Así, se integra sólo para los intervalos de 1s en los que se supera un determinado umbral (generalmente $0.25 \mathrm{~g}$ )

$$
C A V=\sum_{i=1}^{n} \int_{t_{i 0}}^{t_{i 0}+1 s}|a(t)| d t
$$

De este modo, el valor de CAV es nulo para muchos acelerogramas que no superan este umbral. La EPRI considera como umbral de daño en una central nu- 
clear un $C A V=0.3 \mathrm{~g} \cdot \mathrm{s}=2.94 \mathrm{~m} / \mathrm{s}$. La $C A V$ ha mostrado ser un indicador relevante del potencial de daño (EPRI, 1988, Cabañas et al., 1997, Martinez-Rueda \& Tsantali, 2008, Olea et al., 2011), y por tanto de la intensidad sísmica.

Los valores obtenidos de $C A V$ para los 12 sitios analizados (Tabla 1) van desde $291 \mathrm{~cm} / \mathrm{s}$ (min.) en LOR hasta $486 \mathrm{~cm} / \mathrm{s}$ en SP2 (máx.). Los valores de $C A V$ estimados se han representado en la figura 4 y se han comparado con las medias geométricas de $C A V$ obtenidas para un conjunto de terremotos europeos de intensidades VII y VIII (escala EMS).

Duración de la sacudida, $\mathrm{S}_{\mathrm{D}}$. Es claro que la duración del movimiento del terreno tiene influencia en el daño. Una acción sísmica de corta duración sobre un elemento estructural puede mantenerlo en el límite elástico, pero la misma acción repetida un número suficiente de ciclos de deformación, puede provocar el fallo.

Para determinar la duración de forma inequívoca, se han propuesto varias definiciones. Una ampliamente aceptada es debida a Trifunac \& Brady (1975) y se basa en el diagrama de Husid, que es la representación de la función

$$
H(t)=\int_{t_{0}}^{t_{0}+t}|a(\tau)|^{2} d \tau
$$

Esta función crece monótonamente hacia un valor casi asintótico, proporcional a la intensidad de Arias. La duración significativa (significant duration, $\boldsymbol{S}_{\boldsymbol{D}}$ ) se define (Fig. 5) como el intervalo de tiempo entre los instantes en que $\mathrm{H}(\mathrm{t})$ alcanza el $5 \%$ y el $95 \%$ de su valor final. $S_{D}$ crece fuertemente con la magnitud y moderadamente con la distancia.

Los valores de $S_{D}$ obtenidos para los 12 sitios analizados van desde $1 \mathrm{~s}$ (mínimo) en SP1 y un máximo de $1.3 \mathrm{~s}$ en SP2. La breve duración en todos los puntos ha impedido un mayor desarrollo de daños. Estas duraciones son significativamente más cortas que las obtenidas para un conjunto de terremotos europeos de intensidades VII y VIII (escala EMS).

Intensidad espectral, (spectral intensity, $\boldsymbol{S} \boldsymbol{I}$ ). Fue propuesta por Housner (1952), modificando la idea de Benioff (1934) de usar el área bajo la curva del espectro de respuesta de velocidad. Housner definió la intensidad espectral (o de Housner, $\left.I_{H}\right)$ como:

$$
I_{H}=\int_{0.1 s}^{2.5 s} S_{V} d T
$$

donde $\mathrm{S}_{\mathrm{V}}$ es el espectro de respuesta de velocidad (ver más adelante) y $\mathrm{T}$ es el periodo. Los límites de la integral que propuso se justifican porque la mayor parte de las estructuras de edificación tienen modos de vibración entre estos periodos propios. Housner sugirió usar un amortiguamiento de 0.2 . 

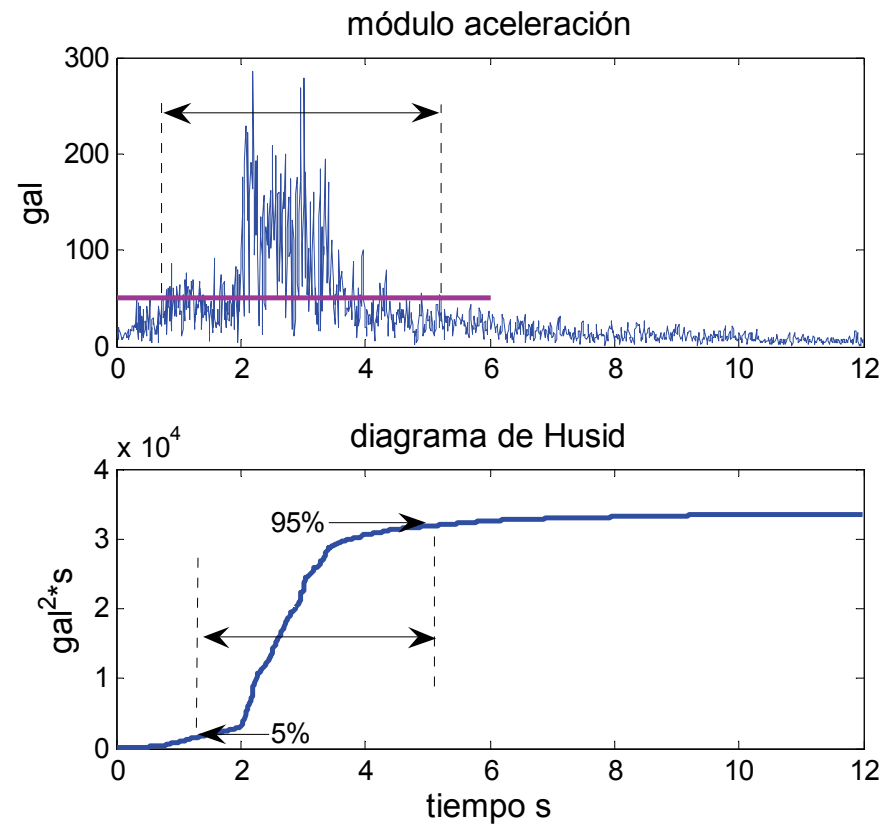

Fig. 5. Cálculo de la duración $B_{D}$ y $S_{D}$. Arriba, la duración válida $B_{D}$ se marca entre los instantes en que se alcanza el umbral de 50 gal por primera y por última vez. Abajo, la duración significativa $S_{D}$, tiempo entre los instantes en los que el diagrama de Husid alcanza el 5\% y el 95\% del valor final.

El uso más frecuente de la intensidad espectral -denominada SI- introduce un factor de normalización dividiendo por el intervalo de periodos de integración, lo que equivale a tomar el valor medio del espectro de respuesta de velocidad en ese rango de periodos (p.e. Yanada et al, 2002).

$$
S I=\frac{1}{\left(T_{2}-T_{1}\right)} \int_{T_{1}}^{T_{2}} S_{V} d T
$$

Aunque los límites de integración propuestos por Housner [0.1, 2.5] pueden no ser completamente representativos y están limitados por la instrumentación de la época (Arias, 1970), siguen siendo los más usados en la medida de $S I$.

Esta escala se refiere al movimiento en un eje particular, aunque también es posible tomar el promedio en los dos ejes horizontales. Yanada et al (2002) usan un $S I$ calculado en tiempo real en ocho direcciones horizontales diferentes. Así se ha hecho en este estudio, tomando el valor máximo de los ocho. Los valores de $S I$ obtenidos para los 12 sitios analizados (Tabla 1) van desde $35.9 \mathrm{~cm} / \mathrm{s}$ (min.) en LOR hasta unos $58 \mathrm{~cm} / \mathrm{s}$ en SP6 y SP4 (máx.). Los valores de $S I$ estimados se han representado en la figura 4 y se han comparado con las medias geométricas de $S I$ 
obtenidas para un conjunto de terremotos europeos de intensidades VII y VIII (escala EMS).

\subsection{Parámetros espectrales}

Los parámetros dependientes de la frecuencia más utilizados son los espectros de respuesta y los espectros de input de energía. Ambos se refieren al movimiento de osciladores amortiguados o sistemas de un grado de libertad (SDOF). Estos sistemas representan los diferentes modos de vibración de las estructuras.

El desplazamiento $x(t)$ del SDOF para una aceleración del suelo arbitraria a(t) puede obtenerse mediante la integral de convolución de Duhamel con la respuesta impulsional $\mathrm{h}(\mathrm{t})$ del oscilador amortiguado

$$
x(t)=h(t) * a(t)=\int_{0}^{t} h(t-\tau) a(\tau) d \tau
$$

Espectros de respuesta (response spectra, $\boldsymbol{S} \boldsymbol{A}, \boldsymbol{S} \boldsymbol{V}$ ). Los espectros de respuesta representan los valores pico de una serie de osciladores amortiguados (generalmente el 5\%) de un grado de libertad con periodos propios diferentes. El valor de pico de esta respuesta $|x(t)|_{\max }$ representado como función de la frecuencia propia $f_{n}=\omega_{n} / 2 \pi$ o bien del periodo propio $T_{n}=1 / f_{n}$ es el espectro de respuesta de desplazamiento, $S D$.

Hay varias técnicas que permiten evitar el cálculo de las integrales (8). Una técnica muy eficiente hace uso del hecho de que la función de transferencia es idéntica a la de un filtro de paso-baja de segundo orden. Se puede simplemente aplicar un filtro numérico recursivo con dicha respuesta al acelerograma a(t) y se obtendrá la respuesta $\mathrm{x}(\mathrm{t})$ correspondiente. De igual modo, es también posible calcular los valores exactos de los espectros de respuesta de velocidad $S V$ y aceleración $S A$.

Los espectros de respuesta permiten el cálculo directo de las tensiones máximas en elementos de una determinada estructura para un movimiento del suelo dado. Se utilizan espectros promedio suavizados y normalizados para los códigos de construcción antisísmica (p.e., NCSE-02). Aunque son útiles para especificar el input sísmico, no proveen información sobre la duración de la sacudida (Danciu $\&$ Tselentis, 2007). Los espectros de respuesta de aceleración $(S A)$ obtenidos en los 12 lugares analizados se han representado en la Figura 6.

En la Figura 6 también se han trazado, como referentes, los valores medios de un conjunto de terremotos euro-mediterráneos de intensidades VII y VIII (EMS). Lo más destacable es la diferencia entre el nivel del espectro en LOR (en roca) con los de los otros sitios, destacando los valores máximos a frecuencias de $\sim 0.4$ s en SP4, SP6, SP3 y SP9, y a frecuencias de $\sim 0.15$ s en SP2, SP5, SP6, SP3 y SP10. Aunque el espectro de respuesta tiene el inconveniente de considerar solo el valor máximo de cada oscilador pero no considera el resto del movimiento, si informa de en qué períodos se ejercen las acciones máximas. 


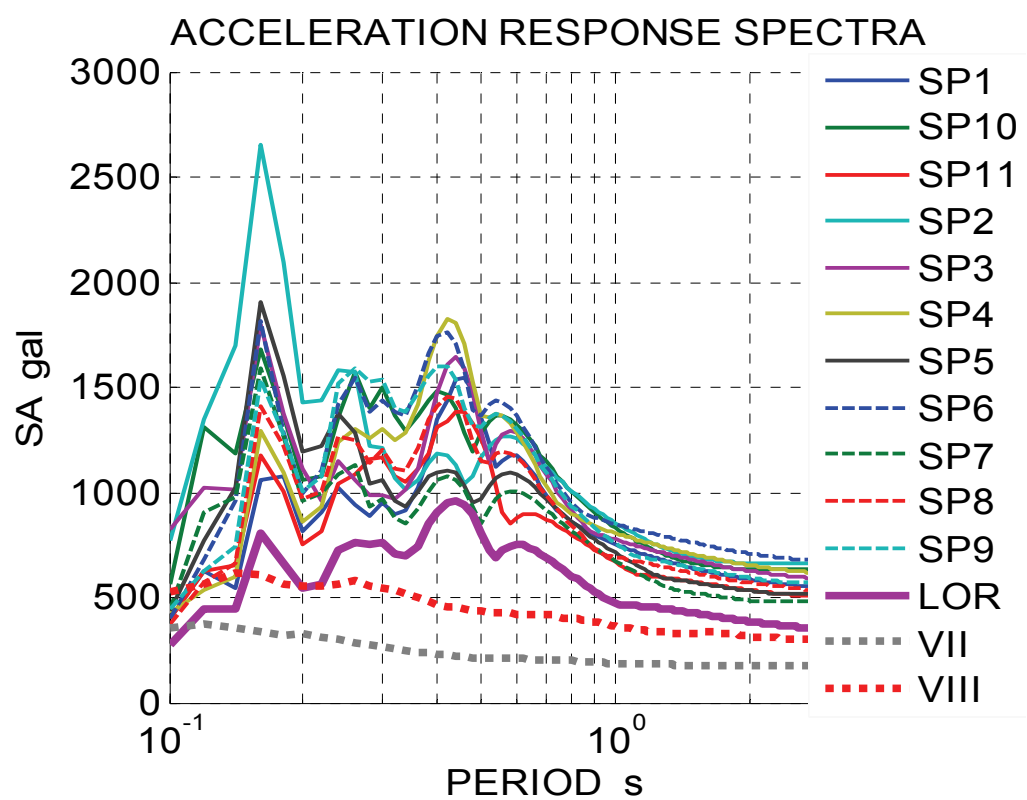

Fig. 6. Espectros de respuesta de aceleración relativa, para un amortiguamiento 5\%, estimados para los 11 puntos con simulación (SP) y para el registro de LOR (roca). También se han trazado, como referencia, los espectros de respuesta esperados obtenidos para terremotos europeos de intensidades locales VII y VIII (EMS).

Los espectros de respuesta de velocidad $(S V)$ obtenidos se han representado en la Figura 7, así como los valores medios correspondientes a los terremotos euromediterráneos mencionados de intensidades VII y VIII (EMS).

Espectro de energía de entrada (Input energy spectrum, IES). Fue introducido por Akiyama (1980), que mostró que la entrada de energía a un edificio por un terremoto es un valor estable, que depende fundamentalmente del periodo libre. Los criterios de diseño basados en energía tienen importancia creciente en la práctica ingenieril y en las normas antisísmicas (p.e. Uang \& Bertero, 1988; Decanini \& Mollaioli, 2001; Benavent-Climent et al., 2002). Si consideramos el trabajo realizado por las fuerzas que aparecen en la ecuación del SDOF,

$$
\int m \ddot{x} d x+\int C \dot{x} d x+\int f(x) d x=-m \int a(t) d x
$$

donde se ha sustituido la fuerza elástica por una expresión más general $f(x)$, que puede tener en cuenta la deformación dúctil. Si no hay deformaciones plásticas, será simplemente $-k x$. 


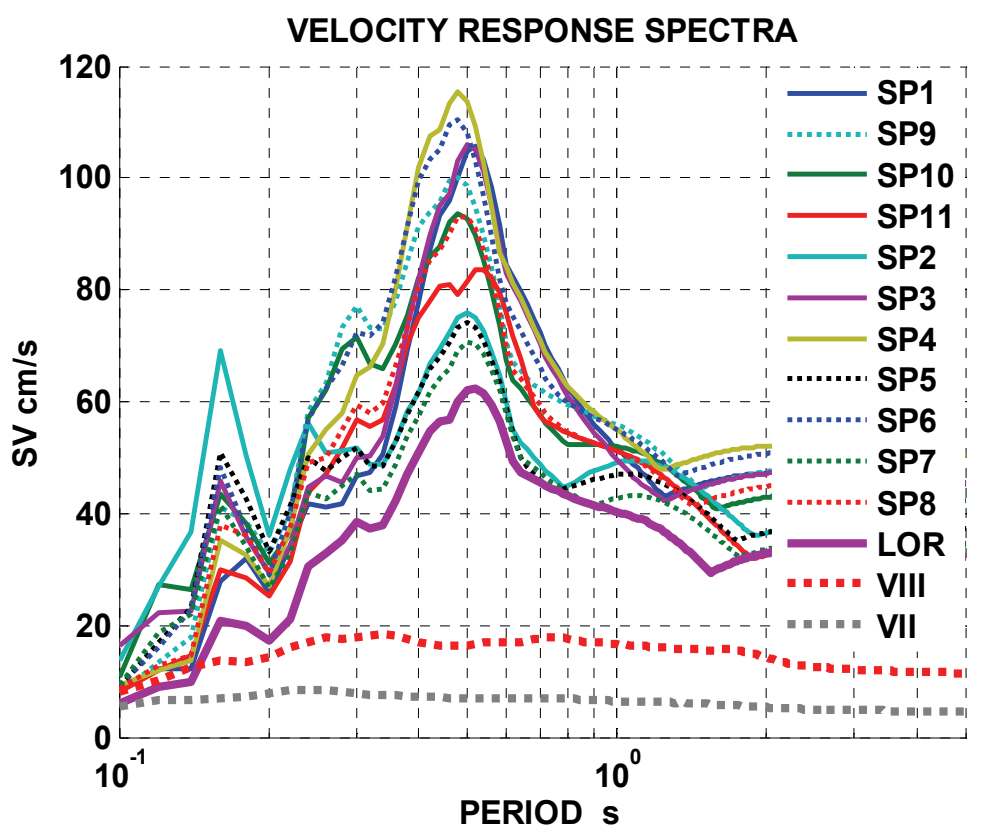

Fig. 7. Espectros de respuesta de velocidad, para un amortiguamiento 5\%, estimados para los 11 puntos con simulación (SP) y para el registro de LOR (roca). También se han trazado, como referencia, los espectros de respuesta medios obtenidos para terremotos europeos de intensidades locales VII y VIII (EMS).

El término del lado derecho de la ecuación es la energía de entrada relativa, que se puede escribir:

$$
E_{i}=-m \int a(t) \dot{x}(t) d t
$$

y representa el trabajo realizado por la fuerza de inercia sobre el SDOF en el SRL, no considerando la traslación como cuerpo rígido con el suelo. La relación $E_{i}$ frente al periodo propio $T_{n}$ es el espectro de input de energía relativa (Uang \& Bertero, 1988). En la práctica se expresa como una pseudo-velocidad $v_{i}$

$$
v_{i}=\sqrt{\frac{2 E_{i}}{m}}=\sqrt{2 \int a(t) \dot{x}(t) d t}
$$

Si consideramos la fuerza recuperadora en el límite elástico, $f(x)=-k x$, puede calcularse el espectro elástico de input de energía dado un amortiguamiento, 
resolviendo primero la ecuación del SDOF para calcular la velocidad $\dot{x}(t)$ de la masa y así aplicar (11) para cada periodo.

Los espectros de energía de entrada (IES) obtenidos se han representado en la Figura 8, así como los valores medios correspondientes a los terremotos euromediterráneos mencionados anteriormente.

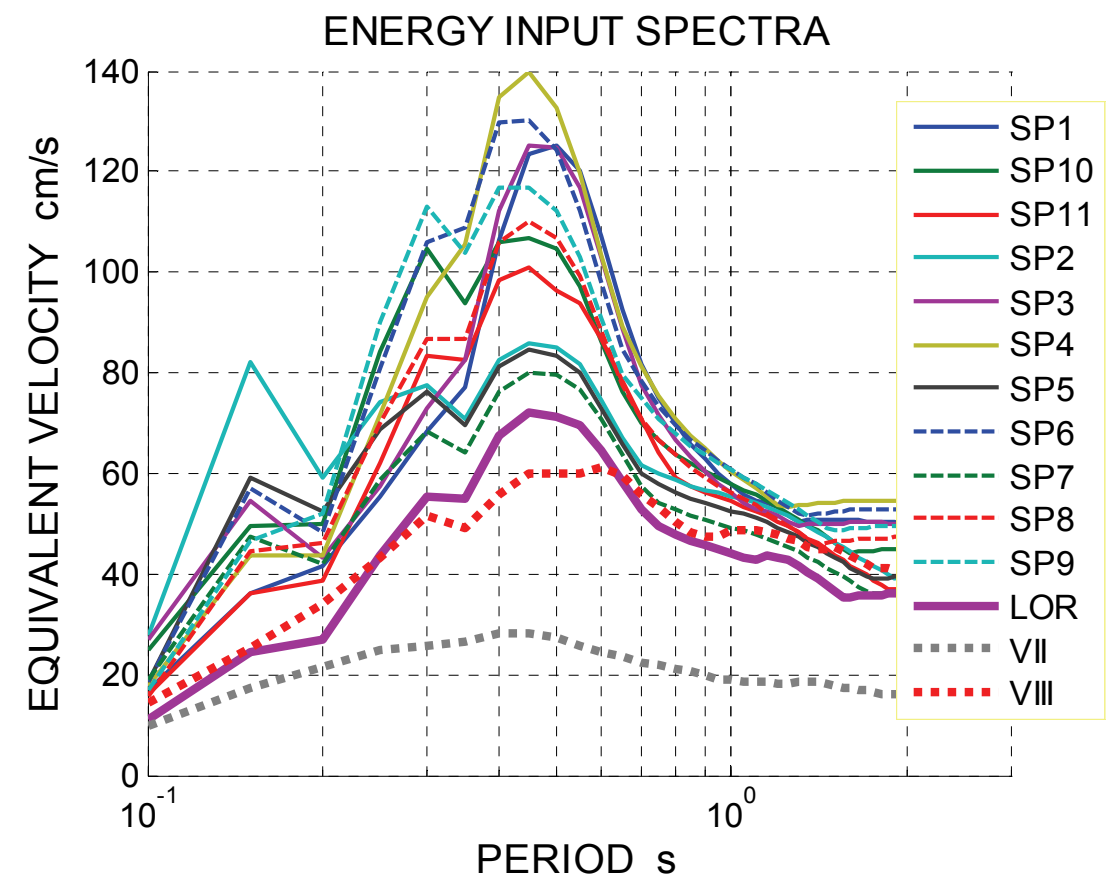

Fig. 8. Espectros de energía de entrada para los de los puntos de simulación (SP) y para el registro de LOR. También se han trazado, como referencia, los espectros de energía de entrada esperados para terremotos europeos de intensidades locales VII y VIII (EMS).

\section{Análisis y discusión de resultados}

Los datos de las estaciones sísmicas de banda ancha temporales desplegadas en el entorno del área fuente de la serie sísmica de Lorca, unidos a los de las estaciones permanentes, han permitido una segunda localización que ha supuesto una mejora importante en la estimación hipocentral de 113 eventos de la serie. Así, la dispersión epicentral que mostraba la localización inicial rutinaria cambió con esta incorporación de estaciones cercanas, ya que los resultados obtenidos muestran un agrupamiento de la distribución espacial en torno a la falla de Alhama de Murcia.

A partir de la anterior localización, se ha aplicado el método de doblediferencia para obtener una relocalización más precisa, que ha agrupado clara- 
mente las réplicas y mostrado una tendencia de dirección NE-SW, que sigue la del rumbo de la falla FAM. Además, la dimensión espacial de la distribución de las réplicas ocurridas los primeros días ha servido para estimar la longitud de la ruptura del evento principal en unos 3.5 a $4 \mathrm{~km}$. Esta dimensión longitudinal coincide aproximadamente con la del diámetro medio de un modelo de fuente circular obtenida a partir de los espectros de la fuente.

La inversiones del tensor momento sísmico de los tres terremotos de mayor magnitud de la serie dan soluciones similares, todas de fallamiento oblicuo, predominantemente inverso con fuerte componente de desgarre, y orientación media de falla en la dirección SW-NE, la misma de la falla FAM.

Las profundidades obtenidas en la relocalización del precursor, principal y réplica mayor son de 5.1, 4.6 y $5.5 \mathrm{~km}$, respectivamente. Esta profundidad coincide con la estimación independiente de la profundidad del centroide, entre 4 y $5 \mathrm{~km}$. Esta profundidad somera y la localización tan cercana a Lorca influyeron en el valor del movimiento del terreno en la ciudad. López-Comino et al. (2012) han analizado las funciones fuente aparentes en diferentes estaciones, poniendo de manifiesto un efecto de directividad hacia Lorca, lo que es otro factor relevante para explicar los altos valores de aceleración y velocidad obtenidos en terreno duro.

Las diferencias geológicas superficiales, evidenciadas con la estructura $\mathrm{V}_{\mathrm{S}}{ }^{30} \mathrm{y}$ con los períodos predominantes del suelo, han influido en la distribución de la intensidad del movimiento del suelo en la ciudad.

Los parámetros instrumentales $P G A, P G V, A I, C A V, S I, S_{D}, S A, S V$ y $I E S$, obtenidos con el acelerograma de la estación LOR y con los movimientos simulados en 11 sitios de la ciudad, a partir de la estructura superficial obtenida con el método SPAC, han mostrado la gran importancia de la geología superficial en las características del movimiento del suelo. Los valores de los parámetros indicados son mayores en todos los sitios simulados respecto a los obtenidos para LOR. Los lugares situados sobre terrenos más blandos y de mayor potencia tienen los valores más altos, destacando el sitio SP6, sobre sedimentos recientes en el valle del Guadalentín.

Los valores de los parámetros $A I, C A V, S I, S A$ y $S V$ son buenos indicadores de la severidad del movimiento del suelo y más representativos de éste que los de $P G A$ y $P G V$. En todos los casos estos valores son superiores a la media geométrica obtenida para sismos euro-mediterráneos de intensidad local VIII (EMS). La duración $S_{D}$ no muestra variaciones apreciables entre los puntos estudiados y sus valores son más cortos que los de referencia. Este parece un punto clave para explicar que, aunque la sacudida puede calificarse de severa en función de los parámetros de fuerte movimiento descritos, los daños que podrían asociarse a una intensidad macrosísmica mayor de la asignada no tuvieron tiempo de desarrollarse más por la corta duración de la sacudida.

Los valores del espectro de respuesta de aceleración relativa ( $S A$ ) muestran valores máximos a $0.15 \mathrm{~s}$ (de hasta más de 2500 gal) y a $0.4 \mathrm{~s}$ (de hasta 1800 gal). Estos valores tan altos son fruto de las amplificaciones debidas al sitio pero 
también a la radiación de la fuente. En los espectros de respuesta de velocidad (SV) destacan los valores altos alrededor de $0.5 \mathrm{~s}$, tanto en roca como en suelo duro, llegando a valores mayores de $110 \mathrm{~cm} / \mathrm{s}$ en los sitios con rellenos más potentes. Los valores más altos en aceleración "espectral" se dan para períodos más cortos $(0.15 \mathrm{~s})$ y los de velocidad para frecuencias más bajas $(0.5 \mathrm{~s})$.

Los valores del espectro de energía de entrada caracterizan mejor el input sísmico en los edificios y por tanto se relacionan adecuadamente con la causa de los daños. Los valores por encima de una velocidad equivalente de $60 \mathrm{~cm} / \mathrm{s}$ se dan para periodos de 0.15 a $0.9 \mathrm{~s}$, y en la mayoría de los sitios analizados presentan valores superiores a $80 \mathrm{~cm} / \mathrm{s}$ para periodos entre $0.25 \mathrm{~s}$ y $0.7 \mathrm{~s}$.

\section{Conclusiones}

La relocalización con estaciones cercanas ha mostrado la importancia de éstas para una localización precisa. El método de doble diferencia ha aportado unos resultados que han deshecho el artefacto creado por las localizaciones de los sismos individuales y ha permitido, además, observar con claridad la tendencia de la distribución espacial de las réplicas en la dirección de la falla FAM.

Los tensores momento sísmico obtenidos muestran un mecanismo focal con un plano acorde con la actividad previa de la falla FAM. Este mecanismo de movimiento oblicuo, similar en los tres eventos importantes, junto con la superficialidad del foco y su cercanía a la ciudad, contribuyen a explicar la sacudida observada, además hay que considerar el efecto de directividad descrito por LópezComino el al. (2012).

Los valores obtenidos de los parámetros $P G A, P G V, A I, C A V$, $S I, S A$ y $S V$ son mayores en todos los puntos simulados que en la estación LOR. Todos ellos son también mayores que los valores medios obtenidos para sismos euromediterráneos de intensidad VIII (EMS), aunque en algún caso están dentro de los márgenes probables para esta intensidad. Sin embargo los $S_{D}$ son excepcionalmente cortos en relación con los valores medios de referencia. Estas cortas duraciones significativas están relacionadas con la baja magnitud del evento y la proximidad epicentral.

Los valores más altos de los espectros de respuesta de aceleración y de velocidad se dan para periodos menores de $0.7 \mathrm{~s}$, con máximos de aceleración en períodos muy bajos $(0.15 \mathrm{~s})$ y los de velocidad en periodos mayores $(0.5 \mathrm{~s})$. Ambos espectros son buenos indicadores del potencial de daño del movimiento en los diferentes emplazamientos.

El espectro de energía de entrada es un parámetro aún más significativo y mejor relacionado con la destructividad de las sacudidas sísmicas en cada lugar. La velocidad equivalente obtenida, con valores superiores a $60 \mathrm{~cm} / \mathrm{s}$ en casi todos los sitios pero en rangos de períodos de diferente ancho, indica qué edificios pueden ser más solicitados. Esto es más relevante en los sitios en los que se superaron $100 \mathrm{~cm} / \mathrm{s}$, con periodos entre $0.3 \mathrm{~s}$ y $0.6 \mathrm{~s}$.

Los factores anteriormente mencionados, junto con las características de la respuesta del terreno explican los valores altos de parámetros del movimiento del 
suelo obtenidos en los diferentes puntos estudiados en Lorca y ponen de manifiesto la gran influencia de las características de la fuente y del sitio en las cercanías de la ruptura.

\section{Agradecimientos}

Este trabajo se ha desarrollado dentro de los proyecto CGL2011-30187-C02-0102 y CGL2008-01830 del Ministerio de Ciencia e Innovación, actualmente Ministerio de Economía y Competitividad, P09-RNM-5100 de la Junta de Andalucía. Financiación adicional ha sido aportada por el proyecto Consolider-Ingenio TOPO-IBERIA (CSD2006-00041). Los autores agradecen los comentarios de un revisor anónimo, que han contribuido a mejorar el manuscrito.

\section{Referencias bibliográficas}

AKIYAMA, H. (1980). Earthquake-resistant limit-state design for buildings. University of Tokyo Press, Tokyo.

ALCALÁ, F. J., NAVARRO, M., GARCÍA-JEREZ, A.,VIDAL, F., CREUS, C. \& ENOMOTO, T. (2012). Geología de la ciudad de Lorca (Murcia, España). Base para evaluar el riesgo sísmico. 7 As. Hispano-Portuguesa de Geodesia y Geofísica. 25-29 Jun., 2012, San Sebastián, 4 pp.

ALGUACIL, G., VIDAL F., NAVARRO, M., GARCÍA-JEREZ, A. \& PÉREZMUELAS, J. (2012). Characterization of earthquake shaking severity in the town of Lorca during the May 11, 2011 event. Bulletin of Earthquake Engineering (en revisión). $15 \mathrm{pp}$.

ALGUACIL G. \& VIDAL, F. (2012). Medidas instrumentales de la intensidad del movimiento del suelo. Aplicación a terremotos europeos, Libro homenaje a la prof. $M^{a}$ Dolores Romacho. Univ. Almeria, 21 pp (en prensa).

AMBRASEYS, N.N., (1975).Trends in engineering seismology in Europe. Proceedings of Fifth European Conference on Earthquake Engineering, vol. 3, pp. $39-52$.

AMBRASEYS, N.N., (1995). The prediction of earthquake peak ground acceleration in Europe. Earthquake Engineering and Structural Dynamics, 24 (4), 467-490.

AMBRASEYS, N.N., DOUGLAS, J., SARMA, S.K. \& SMIT, P.M. (2005). Equations for the Estimation of strong Ground Motions from Shallow Crustal Earthquakes Using Data from Eaurope and the Middle East: Horizontal Peak Ground Acceleration and Spectral Acceleration. Bulletin of Earthquake Engineering 3, 1-53.

ANDERSON J.G., TIBULEAC, I., ANOOSHEHPOOR, A., BIASI, G., SMITH, K. \& VON SEGGERN, D. (2009). Exceptional Ground Motions Recorded during the 26 April $2008 \mathrm{Mw} 5.0$ Earthquake in Mogul, Nevada. Bull. Seism. Soc. Am. 99, 3475-3486,

ANDERSON J.G. (2010). Source and Site Characteristics of Earthquakes That Have Caused Exceptional Ground Accelerations and Velocities. Bull. Seismol. Soc. Am., 100, 1-36, 
ARIAS, A., (1970). A measure of earthquake intensity. In: R.Hansen (Editor), Seismic Design for Nuclear Power Plant. MIT Press, Cambridge, Massachusetts.

BASHAM, P. W., WEICHERT, D.H., ANGLIN, F.M. \& BERRY, M.J.. (1985). New probabilistic strong ground motion maps of Canada, Bull. Seism. Soc. Am. 75, 563-595.

BENAVENT-CLIMENT, A., PUJADES, L.G. \& LÓPEZ-ALMANSA, F. (2002) Design energy input spectra for moderate-seismicity regions. Earthquake Eng. Struct. Dyn. 31, 1151-1172.

BENIOFF, H. (1934). The physical evaluation of seismic destructiveness. Bull. Seism. Soc. Am. 24, 398-403.

BOMMER, J. J. \& ALARCÓN J.E. (2006). The prediction and use of peak ground velocity, J. Earthquake Eng. 10, no. 1, 1-17.

BOORE, D.M. \& JOYNER, W.B. (1982). The empirical prediction of ground motion. Bull. Seism. Soc. Am. 72, 843-860.

BRADLEY, B.A. \& CUBRINOVSKI, M. (2011). Near-source Strong Ground Motions Observed in the 22 February 2011 Christchurch Earthquake. Seismological Reseach Letters 82, 853-865.

BRUNE, J.N. (1970). Tectonic stress and the spectra of the seismic shear waves from earthquakes. J. Geophys.Res.: 75, 4997-5009.

CABAÑAS, L., BENITO, B. \& HERRÁIZ, M. (1997). An approach to the measurement of the potential structural damage of earthquake ground motion. Earthquake Eng. Struct. Dyn. 26, 79-92.

CAMPBELL, K.W. (1981). Near-source attenuation of peak horizontal acceleration. Bull. Seism. Soc. Am. 71, 2039-2070.

CANCANI, A., (1904), "Sur l'emploi d'une double echelle seismique des intesites, empirique et absolue", Gerlands Beitrage Geophysik, 2, 281-283.

DANCIU L. \& TSELENTIS, G.A. (2007). Engineering Ground-Motion Parameters Attenuation Relationships for Greece. Bull. Seism. Soc. Am. 97 (1B), 162183.

DECANINI, L.D. \& MOLLAIOLI, F. (1998).An energy-based methology for the assessment of seismic demand. Soil Dynamics and Earthquake Engineering 21, 113-137.

EC8 - Eurocode 8 EN 1998-1 (2004). Design of structures for earthquake resistance Part 1: General rules, seismic actions and rules for buildings. Commission of the European Communities. Doc CEN/TC250/SC8/N335.

EPRI (1988) A criterion for determining exceedance of the operating basis earthquake. EPRI NP-5930. Electrical Power Research Inst., Palo Alto, CA.

EPRI (1991) Standardization of the cumulative absolute velocity. EPRI TR100082 (Tier 1). Electrical Power Research Inst., Palo Alto, CA.

FAENZA, L. \& MICHELINI, A. (2010) Regression analysis of MCS intensity and ground motion parameters in Italy and its application in ShakeMap. Geophys J Int 180, 1138-1152. 
FEMA Federal Emergency Management Agency (2003). Hazus-MH, FEMA's software program for estimating potential losses from disasters, (último acceso, abril 2012). www.fema.gov/plan/prevent/hazus/index.shtm

HANKS, T.C. \& KANAMORI, H. (1979). A moment magnitude scale. J. Geophys. Res., 84, 2348-2350.

HERSHBERGER, J., (1956),"A comparison of earthquake accelerations with intensity ratings", Bull-of the Seismological Society of America, 46(4), 317320 .

HOUSNER, G.W. (1952). Spectrum Intensities of Strong-Motion Earthquakes. Proc. Symp. Earthquake and Blast Effect on Structures, Earthquake Engineering Research Institute.

HOUSNER, G.W. (1975).Measures of severity of earthquake ground shaking. Proc. of US National Conf. on Earthquake Engineering. pp 25-33.. EERI, Oatkland, CAL, 1975

HUERTA, B. \& REINOSO, E. (2002). Espectros de energía de movimientos fuertes registrados en México. Rev. de Ingeniería Sísmica, 66, 45-72.

IERVOLINO I., GIORGIO M., GALASSO C. \& MANFREDI G. (2008). Prediction relationships for a vector-valued ground motion intensity measure accounting for cumulative damage potential. $14^{\text {th }}$ World Conference on Earthquake Engineering, 2008, China, 8 pp.

KARIM, K.R. \& YAMAZAKI, F. (2002). Correlation of JMA instrumental seismic intensity with strong motion parameters. Earthquake Eng. Struct. Dyn. 31, 1191-1212.

KOSTADINOV, M. V. \& TOWHATA, I. (2002). Assessment of liquefaction inducing peak ground velocity and frequency of horizontal ground shaking at onset of phenomenon, Soil Dyn. Earthquake Eng. 22, no. 4, 309-322.

LANGSTON, C.A., BARKER, J.S. \& PAVLIN, G.B. (1982). Point-source inversion techniques. Phys. Earth Planet. Int., 30, 228-241.

LÓPEZ-COMINO J.A., MANCILLA, F., MORALES, J. \& STICH, D. (2012). Rupture directivity of the 2011, Mw 5.2 Lorca earthquake (Spain). Geophysical Res. Letters. 39, L04707, doi:10.1029/2011GL050720.

MARGOTTINI, C., MOLIN, D. \& SERVA, L. (1992). Intensity versus ground motion: A new approach using Italian data. Engineering Geology, 33, 45-58.

MARTINEZ-DIAZ J.J. (2002). Stress field variety related to fault interaction in a reverse oblique-slip fault: The Alhama de Murcia fault, Betic Cordillera, Spain. Tectonophysics 356, 291-305, doi: 10.1016/S0040- 1951(02)00400-6.

MARTÍNEZ-RUEDA, J.E. \& TSANTALI, E. (2008). Analysis of the correlation between instrumental intensities of strong earthquake ground motion. Eurodyn 2008. $7^{\text {th }}$ European Conference on Structural Dynamics. Southampton.

MASANA, E., MARTÍNEZ-DÍAZ, J.J., HERNÁNDEZ-HENRILE J.L. \& SANTANACH, P. (2004). The Alhama de Murcia fault (SE Spain), a seismogenic fault in a diffuse plate boundary: implications for the IberoMagrebian region. J. Geophys. Res., 109, B01301, doi: 10.029/2002JB002359. 
MEZCUA, J., HERRAIZ, M. \& BUFORN, E. (1984). Study of the 6 June 1977 Lorca (Spain) earthquake and its aftershock sequence. Bull. Seism. Soc. Am. 74, 167-179.

MORALES, J., CANTAVELLA, J.V., MANCILLA, F., LOZANO, L., STICH, D., HERRAIZ, E., MARTÍN, J.B., LOPEZ-COMINO, J.A. \& MARTÍNEZSOLARES, J. M. (2012) The 2011 Lorca seismic series: Temporal evolution, faulting parameters and hypocentral relocation. Bull Earthquake Eng (sometido)

NAKAMURA, Y., (1989). A method for dynamic characteristics estimations of subsurface using microtremors on the ground surface, Quarterly Report, RTRI, Japan, v. 30, p. 25-33.

NAVARRO, M., GARCÍA-JEREZ A., ENOMOTO, T., VIDAL, F., PEREZRUIZ, J. A. \& IWATATE T. (2005). Study of site effects in Lorca town (Murcia, Spain)- using microtremors and strong motion records, Geophys Res Abstr 7, 05184.

NAVARRO, M., GARCÍA-JEREZ, A., ALCALÁ, F., VIDAL, F. \& ENOMOTO T. (2012a). Local site effect microzonation of Lorca town (southern Spain). Bull Earthquake Eng (en revisión).

NAVARRO, N., GARCÍA-JEREZ J.A., ALCALÁ, F.J., VIDAL, F., ARANDA, C. \& ENOMOTO, T. (2012b). Analysis of site effects, building response and damage distribution observed due to 2011 Lorca, Spain, earthquake. 15 World Conf. on Earthquake Engineering, Lisboa 24-28 Sept., 2012, 10 pp.

NCSE-02 (2002) Normativa de Construcción Sismorresistente Española. Real Decreto 997/2002. Boletín Oficial del Estado No. 244, 11 Octubre, 2002, España.

OLEA, B., ALGUACIL, G., VIDAL, F. \& FERICHE, M. (2011). Parámetros de movimiento intenso y su relación con la intensidad macrosísmica en el área euro-mediterránea. 4 Congreso Nacional de Ingeniería Sísmica. Granada. Mayo 2011.

OMORI, F. (1900). Seismic Experiments on the Fracturing and Overturning of Columns. Publ. Earthquake Invest. Comm. in Foreign Languages, N.4, Tokyo.

ORDAZ, M. \& MONTOYA, C. (2002) Programa DEGTRA A4 versión 4.06. Instituto de Ingeniería, Universidad Nacional Autónoma de México, México.

RUEDA, J., MEZCUA, J., \& GARCÍA-BLANCO, R. M. (2011). Directivity effects of the May 11, 2011 Lorca (Spain) Mw=5.1 earthquake. In: Fall Meeting, $A G U$, San Francisco, Communications. S53B-2277.

SARMA, S.K. (1971). Energy flux of strong earthquakes. Tectonophysics, 11, 159-173.

STICH, D., AMMON, C.J. \& MORALES, J. (2003). Moment tensor solutions for small and moderate earthquakes in the Ibero-Maghreb region. J. Geophys. Res. 108, 2148, doi10.1029/2002JB002057.

STICH, D., SERPELLONI, E., MANCILLA, F. \& MORALES, J. (2006). Kinematics of the Iberia-Maghreb plate contact from seismic moment tensors and GPS observations, Tectonophysics 426, 295-317. 
STICH, D., MARTÍN, R. \& MORALES, J. (2010). Moment tensor inversion for Iberia-Maghreb earthquakes 2005-2008. Tectonophysics 483, 390-398.

TRAVASAROU, T., BRAY, D.J. \& ABRAHAMSON, N.A. (2003). Empirical attenuetion relationship for Arias intensity. Earthquake Eng. Struct. Dyn., 32, 1133-1155.

TRIFUNAC, M.D. \& BRADY, A.G. (1975). A study of the duration of strong earthquake ground motion. Bull. Seism. Soc. Am. 65, 581-626.

TSO, W. K., ZHU, T. J. \& HEIDEBRECHT, A. C. (1992). Engineering implication of ground motion A/V ratios, Soil Dyn. Earthquake Eng. 11, 133-144.

UANG, C.M. \& BERTERO, V.V. (1988) Use of energy as a design criterion in earthquake-resistant design. Report No UBC/EERC-88/18. Earthquake Engineering Research Center. University of California at Berkeley

WALD, D. J., QUITORIANO, V., HEATON, T.H. \& KANAMORI, H. (1999). Relationships between peak ground acceleration, peak ground velocity, and modified Mercalli intensity in California. Earthquake Spectra , 15, 557-564.

WALD, D. J., WORDEN,B.C., QUITORIANO, V. \& PANKOW, K. L, (2005). ShakeMap Manual: Technical Manual, User's Guide, Software Guide http://pubs.usgs.gov/tm/2005/12A01/

YANADA, T., FURUKAWA, H., ICHIDA, S., TAKUBO, K., SHIMIZU, Y., KOGANEMARU, K. \& SUZUKI, T. , (2002). Earthquake sensor. Sensors Update 11, 159:216.

WALDHAUSER, F. \& ELLSWORTH W. (2000): Double-Difference earthquake location algorithm: Method and application to the northern Hayward fault, California Bull. Seism. Soc. Am. 90: 1353-1368.

WALDHAUSER, F. (2001): HypoDD: A computer program to compute doubledifference earthquake locations. U.S. Geol. Surv. Open File Rep. 01-113.

VISSERS R.L.M. \& MEIJNINGER, B.M.L. (2011). The 11 May 2011 earthquake at Lorca (SE Spain) viewed in a structural-tectonic context. Solid Earth Discuss 3, 527-540.

YAMAZAKI, F., \& O. MURAO, (2000). Vulnerability functions for Japanese buildings based on damage data from the 1995 Kobe earthquake, in Implications of Recent Earthquakes on Seismic Risk, A. S. Elnashai and S. Antoniou (Editors), Imperial College Press, 91-102. 